\title{
Lamotrigine as an adjuvant treatment for acute bipolar depression: a Brazilian naturalistic study
}

\author{
Luciana Angélica Silva Silveira ${ }^{1}$, Fernanda Demôro Novis ${ }^{1}$, Rafaela Oliveira da Silva ${ }^{1}$, Ana \\ Letícia Santos Nunes $^{1}$, Pedro Guimarães Coscarelli², and Elie Cheniaux ${ }^{1,2}$ \\ 1- Universidade Federal do Rio de Janeiro, Rio de Janeiro, RJ, Brazil \\ 2- Universidade do Estado do Rio de Janeiro, Rio de Janeiro, RJ, Brazil
}

\begin{abstract}
Lamotrigine is indicated according to several recent treatment guidelines as a first-line medication for the treatment of bipolar depression. However, its efficacy in acute bipolar depression has not been well established. In the present naturalistic study, patients with bipolar depression $(n=20)$, predominantly bipolar type I, were treated with lamotrigine in addition to their prior treatment for 8 weeks. The Young Mania Rating Scale (YMRS), 17-item Hamilton Rating Scale for Depression (HAM-D-17), and Clinical Global Impressions-Bipolar Disorder (CGI-BD) scale were applied at baseline, week 4 , and week 8 . With regard to the primary measure of efficacy, mean total HAM-D-17 scores significantly decreased $(p<.01)$ at the end of treatment. Eight patients $(40 \%)$ exhibited a positive response (i.e., at least a 50\% reduction of baseline scores). Additionally, eight (40\%) and 11 (55\%) patients exhibited complete remission, reflected by HAM-D-17 and CGI-BP scores, respectively. Episodes of switching to mania or hypomania occurred in five patients $(25 \%)$. No skin rash or any other significant adverse events were reported. Our results indicate that the addition of lamotrigine to a mood stabilizer can be useful in the treatment of acute depressive episodes in bipolar I disorder. Keywords: lamotrigine, bipolar disorder, depression, naturalistic study.
\end{abstract}

Received 18 December 2012; received in revised form 11 March 2013; accepted 1 May 2013. Available online 27 June 2013.

\section{Introduction}

Lamotrigine is indicated by at least four recent treatment guidelines as a first-line medication in the treatment of bipolar depression (Nivoli et al., 2011). However, its efficacy in acute bipolar depression has not been well established to date. In five randomized, doubleblind, placebo-controlled clinical trials, lamotrigine did not differ from placebo (Calabrese et al., 2008). In a metaanalysis of these same studies that considered only the most severe cases, however, lamotrigine was demonstrated to be effective (Geddes, Calabrese, \& Goodwin, 2009).

Modern,randomized, double-blind, placebo-controlled clinical trials that investigate treatments for bipolar disorder have important methodological limitations such as highly selected samples and high dropout rates (Cheniaux, 2011). Moreover, in contrast to the usual practice of treating

Luciana Angélica Silva Silveira, Fernanda Novis, Rafaela Oliveira da Silva, Ana Letícia Santos Nunes, and Elie Cheniaux, Instituto de Psiquiatria da Universidade Federal do Rio de Janeiro. Pedro Guimarães Coscarelli and Elie Cheniaux, Faculdade de Ciências Médicas da Universidade do Estado do Rio de Janeiro. Correspondence regarding this article should be directed to: Luciana Angélica Silva Silveira, Rua Lauro Müller, 46 / 310 - Botafogo, Rio de Janeiro, RJ, 22290-160, Brazil. Phone: +55 21 9466-1516. E-mail: lucianangelica@ hotmail.com individuals with bipolar disorder, the compound tested in these studies was used as monotherapy. Therefore, direct extrapolation of these results and conclusions to everyday clinical practice becomes questionable (Beutler, 1998). Therefore, naturalistic studies could add to the current knowledge about treating bipolar disorder.

A few naturalistic studies have investigated the use of lamotrigine in bipolar depression. These studies generated consistent results with lamotrigine showing effectiveness across studies. Nevertheless, these studies diverged with regard to the sample composition. Two Canadian studies included patients with bipolar disorder not otherwise specified with refractory depression (Kusumakar \& Yatham, 1997) and bipolar II resistant to treatment (Sharma, Khan, \& Corpse, 2008). Two American studies included patients with a mixture of bipolar I and II disorder and schizoaffective disorder (Ketter et al., 2008) and bipolar I and II disorder (Joe, Chang, Won, Rim, Ha, \& Ha, 2009). A Korean study included patients with only bipolar II with a partial response to other mood stabilizers (Chang, Moon, Cha, \& Ha, 2010).

We conducted a naturalistic study of the use of lamotrigine as an adjuvant treatment in adult Brazilian patients with bipolar disorder, predominantly bipolar I, during an acute depressive episode. 
Methods

\section{Subjects}

The present study was conducted in a research outpatient clinic for bipolar disorder at the Institute of Psychiatry, Federal University of Rio de Janeiro (UFRJ), Brazil. The project was approved by the local ethics committee. Patients or their legal guardians provided written informed consent.

The study comprised all patients diagnosed with bipolar disorder who had a depressive episode and were treated with lamotrigine at a minimum dose of 25 $\mathrm{mg}$ /day for at least 8 weeks. Clinical assessment was performed prospectively.

\section{Clinical instruments}

The diagnoses of bipolar disorder and comorbidities were established using the Structured Clinical Interview for Diagnostic and Statistical Manual of Mental Disorders, 4th edition (DSM-IV), Disorders (SCID; DelBen, Vilela, de Crippa, Hallak, Labate, \& Zuardi, 2010). Sociodemographic data for each patient were recorded. A clinical history was taken including age of disease onset and number of previous hospitalizations, affective episodes, and suicide attempts. At baseline and weeks 4 and 8 of treatment, three clinical evaluation scales were administered: the Young Mania Rating Scale (YMRS; Young, Biggs, Ziegler, \& Meyer, 1978), the 17-item Hamilton Rating Scale for Depression (HAM-D-17; Hamilton, 1960), and the Clinical Global ImpressionsBipolar Disorder (CGI-BD) scale (Spearing, Post, Leverich, Brandt, \& Nolen, 1997).

\section{Procedure}

Inclusion criteria were the following: 18 years of age or older, DSM-IV-TR (American Psychiatric Association, 2000) diagnosis of bipolar I or II disorder, total baseline HAM-D-17 score $>8$, CGI-BP score for depression $>2$, total YMRS score $\leq 12$, and CBI-BP score for mania $<3$.

Exclusion criteria were the following: refusal to cooperate in completing the assessment instruments, presence of severe non-psychiatric disease, and use of antidepressants during the evaluation period or during the 4 weeks preceding the introduction of lamotrigine. Patients who used other psychotropics concomitantly, with the exception of antidepressants, were not excluded. Those patients with a psychiatric comorbidity were also not excluded.

Patients who met the aforementioned inclusion criteria received an initial dose of $25 \mathrm{mg}$ lamotrigine, which was escalated by $25 \mathrm{mg}$ every 2 weeks to prevent skin rashes, a potential risk for progressing to StevensJohnson syndrome. In the case of the concomitant use of valproic acid, the dose was escalated at a slower pace (i.e., every 4 weeks). The maximum doses of lamotrigine for each patient were individually chosen according to clinical criteria.
The HAM-D-17 score was chosen as the primary outcome measure. The total scores at baseline were compared with the scores obtained at week 8 of lamotrigine treatment. Baseline and final HAM-D-17 scores for items $1,3,7$, and 17 were used as secondary outcome measures and compared. These items evaluate, respectively, depressed mood, suicidal ideation, functional capacity, and awareness of illness, aspects that we believe are particularly relevant in the context of depression. Item 1 was evaluated individually in five double-blind studies, and item 17 was assessed in three of those studies (Calabrese et al., 2008). Furthermore, the number of patients who responded positively to treatment, defined as at least a $50 \%$ reduction of the total HAM-D-17 score, was also studied. Additionally, the rates of remission defined as either a HAM-D-17 score $\leq 8$ at week 8 or CGI-BP score for depression $\leq 2$ were investigated. Lastly, the rates of switching to mania or hypomania were recorded, defined as either a YMRS score $>12$ or CGI-BP score for mania $>2$ at any point during the evaluation.

\section{Statistical analysis}

To compare the scores at baseline and week 8 after lamotrigine was initiated, we used the Wilcoxon test for two paired samples. For the analysis of associations between a positive response to lamotrigine and binary variables (i.e., previous suicide attempt, ultradian cycling, and use of other medications), Fisher's test was used. For the continuous variables (i.e., age of onset, time of bipolar disorder, number of previous hospitalizations, number of depressive episodes, and number of manic episodes), logistic regression models were applied. All of the analyses were conducted using $\mathrm{R}$ software, the respective script for which is available from the corresponding author.

\section{Results \\ Sample description}

Twenty bipolar patients in a current depressed mood fulfilled the inclusion criteria and were treated with lamotrigine in association with their previous medication. Table 1 presents the main demographic and clinical aspects of the patients. Of the 20 patients, 17 had bipolar I and three patients had bipolar II. Ages of the patients ranged from 21 to 70 years (mean, 45.1 years; standard deviation $[S D]=12.2)$. Only two patients were male.

The minimum dose of lamotrigine was $25 \mathrm{mg}$ daily, and the maximum was $200 \mathrm{mg}$ (mean, $75.96 \mathrm{mg}$ ). Three patients used $25 \mathrm{mg}$, seven used $50 \mathrm{mg}$, one used $75 \mathrm{mg}$, eight used $100 \mathrm{mg}$, and one used $200 \mathrm{mg}$. The following drugs were used in association with lamotrigine: lithium (18 patients), carbamazepine (six patients), valproic acid (one patient), antipsychotics (i.e., thioridazine, sulpiride, risperidone, and olanzapine; seven patients), benzodiazepines (i.e., clonazepam, diazepam, and lorazepam; 14 patients), and levothyroxine (two patients). 
Table 1. Demographic and clinical variables

\begin{tabular}{ll}
\hline Age (years [median]) & $45.1(\mathrm{SD}=12.2)$ \\
Gender (female/male) & $17 / 3$ \\
Bipolar disorder (type I/type II) & $17 / 3$ \\
Depression level (mild/moderate/marked) & $5 / 9 / 6$ \\
Age of illness onset (years [median]) & $22.35(\mathrm{SD}=12.46)$ \\
Number of previous hospitalizations & \\
0 & 9 \\
1 & 4 \\
2 & 3 \\
3 & 3 \\
4 & 1 \\
DSM-IV Axis I comorbidity & \\
None & 8 \\
Panic disorder & 5 \\
Social phobia & 5 \\
Specific phobia & 5 \\
Obsessive-compulsive disorder & 4 \\
Alcohol-related disorders & 3 \\
Anorexia nervosa & 2 \\
Posttraumatic stress disorder & 1 \\
Lamotrigine dosage (mg [median]) & $75.96(\mathrm{SD}=42.28)$ \\
Medications concomitant with lamotrigine & \\
None & 0 \\
Lithium & 18 \\
Carbamazepine & 6 \\
Valproic acid & 1 \\
Antipsychotics & \\
Benzodiazepines & \\
Levothyroxine & \\
\hline
\end{tabular}

\section{Efficacy and tolerability of lamotrigine}

Table 2 presents the main results. With regard to efficacy, the primary outcome measure, the mean total HAM-D-17 scores significantly decreased $(p<.01)$ from $15.15(S D=5.42)$ at baseline to $9.1(S D=4.78)$ at week 8 of lamotrigine treatment. According to the HAM-D-17, eight of the 20 patients showed a positive response (i.e., at least a $50 \%$ reduction of the total scores at the endpoint of the study). Six of these patients and two others were

Table 2. Main results related to treatment with lamotrigine $(n=20)$

\begin{tabular}{|c|c|}
\hline Baseline HAM (total score [median]) & $15.15(\mathrm{SD}=5.42)$ \\
\hline Final HAM (total score [median]) & $9.1(\mathrm{SD}=4.78)$ \\
\hline $\begin{array}{l}\text { Positive response ( } 50 \% \text { reduction of HAM } \\
\text { score) (number of patients) }\end{array}$ & 8 \\
\hline Remission in HAM (number of patients) & 8 \\
\hline Remission in CGI-BP (number of patients) & 11 \\
\hline Switching in YMRS (number of patients) & 5 \\
\hline Switching in CGI-BP (number of patients) & 5 \\
\hline
\end{tabular}

considered to have achieved complete remission of their depressive symptoms. With regard to specific HAM-D-17 items, a statistically significant reduction was found in the mean scores for item 1 (from $2.55[S D=.99]$ to 1.2 $[S D=1.15] ; p<.01$ ), item 3 (from $1.2[S D=1.19]$ to .3 $[S D=.73] ; p<.05$ ), and item 7 (from $2.25[S D=1.02]$ to $.85[S D=1.04] ; p<.01)$. However, for item 17, the mean score reduction from $.15(S D=.49)$ to $.0(S D=0)$ was not significant $(p=.37)$.

The mean CGI-BP scores for depression significantly decreased $(p<.001)$ from $4.05(S D=.76)$ at baseline to $2.3(S D=1.38)$ at week 8 . Based on the CGI-BP, 11/20 patients had complete remission of their depressive symptoms.

Episodes of switching to mania or hypomania after the introduction of lamotrigine occurred in five of the 20 patients, reflected by YMRS and CGI-BP scores. No association was found between a positive response with lamotrigine and any retrospective clinical data such as age of bipolar disorder onset, time of disease, number of hospitalizations, suicide attempts, previous affective episodes, or history of ultradian cycling. Likewise, no association was found between a positive response and the concomitant use of any medication with lamotrigine.

\section{Adverse effects of lamotrigine}

No skin rashes or any other significant adverse events were reported. All patients completed the study.

\section{Discussion}

The present study evaluated lamotrigine as an adjuvant treatment for bipolar depression in a sample of 20 patients and found a statistically significant overall reduction of depressive symptoms after 9 weeks of treatment. Eight patients $(40 \%)$ exhibited a positive response (i.e., at least a $50 \%$ reduction of baseline scores). Additionally, eight (40\%) and 11 (55\%) patients exhibited complete remission, reflected by HAM-D-17 and CGI-BP scores, respectively. No retrospective clinical data or concomitant medication was related to a positive response with lamotrigine.

Treatment for bipolar depression has been much less often studied than the treatment of unipolar depression and mania (Goodwin, Jamison, \& Ghaemi, 2007). To date, the U.S. Food and Drug Administration has only approved two agents for the treatment of bipolar depression: quetiapine and the combination of fluoxetineolanzapine. Although lamotrigine has been approved solely for the prevention of new affective episodes in the therapy of bipolar disorder, some treatment guidelines (Yatham et al., 2009) place lamotrigine as a first-line option in treating acute bipolar depression.

In the first large placebo-controlled study of the use of lamotrigine in bipolar depression, this compound was superior to placebo only in secondary measures of efficacy (Calabrese, Bowden, Sachs, Ascher, Monaghan, $\&$ Rudd, 1999). In four other similar studies, the results of which were published together, lamotrigine was not 
different from placebo in any of the efficacy measures assessed (Calabrese et al., 2008). However, a metaanalysis of these five studies (Geddes et al., 2009) indicated that a significantly greater number of patients responded to lamotrigine than to placebo and that lamotrigine was more effective in a subgroup of patients with higher scores on scales of depressive symptoms.

Consistent with the present results, five other naturalistic studies suggested the value of using lamotrigine as an adjuvant treatment for bipolar depression. In one study, 22 subjects with bipolar disorder (n.b., the type was not specified) participated. These patients had depression that was refractory to the combination of sodium divalproate and another stabilizer or antidepressant. Patients were treated for 6 weeks with lamotrigine as an add-on to the previous medication and evaluated on a weekly basis using the HAM-D. At the end of week $6,63 \%$ of the subjects achieved remission (Kusumakar \& Yatham, 1997). In a retrospective naturalistic study, the sample consisted of 31 patients with bipolar II disorder who had depression that was resistant to treatment with a combination of two stabilizers or a mood stabilizer and antidepressant. Lamotrigine was used alone or combined with other psychotropics for 6 months. Upon the completion of follow-up, the use of lamotrigine was found to be associated with significant improvement in $52 \%$ of the cases. Additionally, after the 6-month period, $45 \%$ of the patients no longer fulfilled the DSM-IV criteria for a major depressive episode (Sharma et al., 2008).

In another naturalistic study, the sample was markedly heterogeneous and included patients with bipolar I disorder, bipolar II disorder, and bipolar disorder not otherwise specified and patients with schizoaffective disorder. Considering exclusively the 53 patients who were initially depressed, the addition of lamotrigine in association with the previous medication led to complete remission in 16 patients $(30.2 \%)$. Moreover, 25 patients $(47.16 \%)$ were rid of depression according to the DSMIV-TR criteria (Ketter et al., 2008).

Two other naturalistic studies were conducted in South Korea. The first focused on lamotrigine dose titration in bipolar depression. A total of 259 patients were allocated to two groups: one group followed the standard dose escalation for lamotrigine, and the other group followed a slower schedule. A statistically significant reduction of CGI-BP scores was found over the course of 12 weeks in both groups, suggesting the clinical efficacy of lamotrigine treatment (Joe et al., 2009). The second study included a sample of 109 patients diagnosed with bipolar II disorder with no Axis I comorbidities who were in a current depressive episode and had not responded adequately to other mood stabilizers. These patients were given lamotrigine as an adjuvant treatment and followed for 52 weeks. A significant reduction of CGI-BP depression scores was observed as early as week 4 , which was sustained throughout the follow-up period. The response rate at the end of week 12 was $64.2 \%$ (Chang et al., 2010).
Open-label studies of lamotrigine in the treatment of bipolar depression for specific populations have also been conducted. The sample sizes of these studies were variable: five geriatric patients (Robillard \& Conn, 2002), 20 adolescents (Chang, Saxena, \& Howe, 2006), and 57 elderly patients (Sajatovic et al., 2011). The first study failed to specify the type of bipolar disorder, and the other two studies included bipolar I and II patients. All three studies found a statistically significant reduction of depression scores on their assessment scales.

One alternative to monotherapy with lamotrigine in bipolar depression is to use it as an adjuvant treatment. In a randomized double-blind study, the combination of lithium and lamotrigine was superior to the combination of lithium and placebo, considering both the global reduction of Montgomery-Åsberg Depression Rating Scale scores and the number of cases that had a positive response (van der Loos et al., 2009).

In the present study, several patients showed a good response to very low doses of lamotrigine (i.e., $<100 \mathrm{mg}$ /day). This may have occurred in some cases because of a placebo effect, which cannot be dismissed in naturalistic studies. However, lamotrigine as an adjuvant treatment may be administered at lower doses than those prescribed for monotherapy in the treatment of acute bipolar depression. To the best of our knowledge, a dearth of studies have determined the doses of lamotrigine that should be prescribed when associated with other medications such as other mood stabilizers or atypical antipsychotics.

The rate of manic or hypomanic switching was high in the present study, corresponding to $25 \%$ of the patients. In fact, some case reports described an association between the use of lamotrigine and switching to mania or hypomania (Cheniaux, Dias, Lessa, \& Versiani, 2005). However, in contrast to our results, a naturalistic study of lamotrigine treatment for bipolar depression (Kusumakar \& Yatham, 1997) found no manic or hypomanic switching, and another study noted a switching rate of only 3.8\% (Ketter et al., 2008). A 1-year, open-label study of lamotrigine as maintenance treatment after an episode of bipolar depression found a lower rate of switching compared with the preceding year (McElroy et al., 2004). Lastly, a randomized, double-blind study found that only $5.4 \%$ of the patients who took lamotrigine exhibited manic or hypomanic switching compared with $4.6 \%$ of the patients who took placebo. The difference was not statistically significant (Calabrese et al., 1999).

Synergistic or additive effects between lamotrigine and other stabilizers or atypical antipsychotics are believed to occur through serotonergic mechanisms, monoaminergic activation, or the modulation of sodium channels, which would account for the action of lamotrigine as an adjuvant treatment in bipolar depression (Consoni, Vital, \& Andreatini, 2006; Prica, Hascoet, \& Bourin, 2008; Bourin, Chenu, \& Hascoët, 2009; van der Loos et al., 2009).

Although the present results indicate the efficacy of lamotrigine in acute bipolar depression, a few 
limitations that are basically inherent to naturalistic studies should be mentioned. The sample was quite small and heterogeneous, with a high rate of psychiatric comorbidities. Additionally, most of our patients came from a teaching hospital service. For this reason, they may have presented with a greater level of severity than other bipolar disorder patients. No comparisons with placebo were made. Several concomitant medications were used, especially mood stabilizers, but the use of several antipsychotics was also reported, thus hampering the ability to draw definitive conclusions about the effects of lamotrigine specifically. The doses of these associated medications were changed over the 8 weeks of evaluation and some were discontinued, which may have influenced the final response. Finally, because nearly all of the patients in our sample had bipolar I, our results cannot be extrapolated to bipolar II disorder. Therefore, further controlled studies with larger samples should be conducted to elucidate the efficacy of lamotrigine in managing the acute phase of bipolar depression, not only as monotherapy but also as an adjuvant treatment.

The efficacy of monotherapy lamotrigine for the treatment of bipolar depression has not been well established, but the present results indicate that the addition of lamotrigine to a mood stabilizer may be useful in the treatment of an acute depressive episode in bipolar I disorder.

\section{References}

American Psychiatric Association (2000). Diagnostic and statistical manual of mental disorders, 4th edition, text revision. Washington, DC: American Psychiatric Press.

Beutler, L. E. (1998). Identifying empirically supported treatments: what if we didn't? Journal of Consulting and Clinical Psychology, $66,113-120$

Bourin, M., Chenu, F., \& Hascoët, M. (2009). The role of sodium channels in the mechanism of action of antidepressants and mood stabilizers. Current Drug Targets, 10, 1052-1060.

Calabrese, J. R., Bowden, C. L., Sachs, G. S., Ascher, J. A., Monaghan, E., \& Rudd, G. D. (1999). A double-blind placebo-controlled study of lamotrigine monotherapy in outpatients with bipolar I depression. Journal of Clinical Psychiatry, 60(2), 79-88.

Calabrese, J. R., Huffman, R. F., White, R. L., Edwards, S., Thompson, T. R., Ascher, J. A., Monaghan, E. T., \& Leadbetter, R. A. (2008). Lamotrigine in the acute treatment of bipolar depression: results of five double-blind, placebo-controlled clinical trials. Bipolar Disorder, 10, 323-333.

Chang, K., Saxena, K., \& Howe, M. (2006). An open-label study of lamotrigine adjunct or monotherapy for the treatment of adolescents with bipolar depression. Journal of the American Academy of Child and Adolescent Psychiatry, 45(3), 298-304.

Chang, J. S., Moon, E., Cha, B., \& Ha, K. (2010). Adjunctive lamotrigine therapy for patients with bipolar II depression partially responsive to mood stabilizers. Progress in Neuropsychopharmacology and Biological Psychiatry, 34(7), 1322-1326.

Cheniaux, E., Dias, A., Lessa, J. L. M., \& Versiani, M. (2005). Can lamotrigine induce a switch into mania? Revista de Psiquiatria do Rio Grande do Sul, 27(2), 206-209.

Cheniaux, E. (2011). The pharmacological treatment of bipolar disorder: A systematic and critical review of the methodological aspects of modern clinical trials. Revista Brasileira de Psiquiatria, 33(1), 72-80.

Consoni, F. T., Vital, M. A., \& Andreatini, R. (2006). Dual monoamine modulation for the antidepressant-like effect of lamotrigine in the modified forced swimming test. European Neuropsychopharmacology, 16, 451-458.

Del-Ben, C. M., Vilela, J. A. A., de Crippa, J. A. S., Hallak, J. E. C., Labate, C. M., \& Zuardi, A. W. (2010). Confiabilidade da "Entrevista Clínica Estruturada para o DSM-IV: Versão Clínica" traduzida para o Português. [Reliability of the Structured Clinical Interview for DSM-IV: Clinical version; translated into Portuguese]. Revista Brasileira de Psiquiatria, 23(3), 156-159.

Geddes, J. R., Calabrese, J. R., \& Goodwin, G. M. (2009). Lamotrigine for treatment of bipolar depression: Independent meta-analysis and meta-regression of individual patient data from five randomised trials. British Journal of Psychiatry, 194, 4-9.

Goodwin, F. K., Jamison, K. R., \& Ghaemi, S. N. (2007). Manicdepressive illness: Bipolar disorders and recurrent depression, 2nd edition (p. 841). New York: Oxford University Press.

Hamilton, M. (1960). A rating scale for depression. Journal of Neurology, Neurosurgery, and Psychiatry, 23, 56-62.

Joe, S. H., Chang, J. S., Won, S., Rim, H. D., Ha, T. H., \& Ha, K. (2009). Feasibility of a slower lamotrigine titration schedule for bipolar depression: a naturalistic study. International Clinical Psychopharmacology, 24(2), 105-110.

Ketter, T. A., Brooks, J. O., Hoblyn, J. C., Champion, L. M., Nam, J. Y., Culver, J. L., Marsh, W. K., \& Bonner, J. C. (2008). Effectiveness of lamotrigine in bipolar disorder in a clinical setting. Journal of Psychiatric Research, 43, 13-23.

Kusumakar, V., \& Yatham, L. N. (1997). An open study of lamotrigine in refractory bipolar depression. Psychiatry Research, 72(2), 145-148.

McElroy, S. L., Zarate, C. A., Cookson, J., Suppes, T., Huffman, R. F., Greene, P., \& Ascher, J. (2004). A 52-week, open-label continuation study of lamotrigine in the treatment of bipolar depression. Journal of Clinical Psychiatry, 65, 204-210.

Nivoli, A. M., Colom, F., Murru, A., Pacchiarotti, I., Castro-Loli, P., González-Pinto, A., Fountoulakis, K. N., \& Vieta, E. (2011). New treatment guidelines for acute bipolar depression: A systematic review. Journal of Affective Disorders, 129(1-3), 14-26.

Prica, C., Hascoet, M., \& Bourin, M. (2008). Antidepressant-like effect of lamotrigine is reversed by veratrine: A possible role of sodium channels in bipolar depression. Behavioural Brain Research, 191, 49-54.

Robillard, M., \& Conn, D. K. (2002). Lamotrigine use in geriatric patients with bipolar depression. Canadian Journal of Psychiatry, 47(8), 767-770.

Sajatovic, M., Gildengers, A., Al Jurdi, R. K., Gyulai, L., Cassidy, K. A., ...Young, R. C. (2011). Multisite, open-label, prospective trial of lamotrigine for geriatric bipolar depression: A preliminary report. Bipolar Disorder, 13(3), 294-302.

Sharma, V., Khan, M., \& Corpse, C. (2008). Role of lamotrigine in the management of treatment resistant bipolar II depression: A chart review. Journal of Affective Disorders, 111, 100-105.

Spearing, M. K., Post, R. M., Leverich, G. S., Brandt, D., \& Nolen, W. (1997). Modification of the Clinical Global Impressions (CGI) Scale for use in bipolar illness (BP): The CGI-BP. Psychiatry Research, 73(3), 159-171.

van der Loos, M. L., Mulder, P. G., Hartong, E. G., Blom, M. B., Vergouwen, A. C., de Keyzer, H. J., ... Nolen, W. A. (2009). Efficacy and safety of lamotrigine as add-on treatment to lithium in bipolar depression: A multicenter, double-blind, placebo-controlled trial. Journal of Clinical Psychiatry, 70, 223-231.

Yatham, L. N., Kennedy, S. H., Schaffer, A., Parikh, S. V., Beaulieu, S., O’Donovan, C., ... Kapczinski, F. (2009). Canadian Network for Mood and Anxiety Treatments (CANMAT) and International Society for Bipolar Disorders (ISBD) collaborative update of CANMAT guidelines for the management of patients with bipolar disorder: update 2009. Bipolar Disorder, 11, 225-255.

Young, R. C., Biggs, J. T., Ziegler, V. E., \& Meyer, D. A. (1978). A rating scale for mania: Reliability, validity and sensitivity. British Journal of Psychiatry, 133, 429-435. 
